\title{
Adipocyte miR-200b/a/429 ablation in mice leads to high-fat- diet-induced obesity
}

\author{
Cong Tao ${ }^{1,2, *}$, Hongyan Ren ${ }^{1,3, *}$, Pan Xu ${ }^{1}$, Jia Cheng ${ }^{1}$, Sujuan Huang ${ }^{1}$, Rong Zhou ${ }^{1}$, \\ Yulian Mu ${ }^{1}$, Shulin Yang ${ }^{1}$, Desheng $\mathbf{Q i}^{2}$, Yanfang Wang ${ }^{1}$ and ${\mathrm{Kui} \mathbf{L i}^{1}}^{1}$ \\ ${ }^{1}$ State Key Laboratory of Animal Nutrition, Institute of Animal Science, Chinese Academy of Agricultural Sciences, Beijing, \\ China \\ ${ }^{2}$ College of Animal Science and Technology, Huazhong Agricultural University, Wuhan, China \\ ${ }^{3}$ Key Laboratory of Animal Embryo \& Molecular Breeding of Hubei Province, Institute of Veterinary and Animal Science, \\ Hubei Academy of Agricultural Science, Wuhan, China \\ * These authors have contributed equally to this work \\ Correspondence to: Kui Li, email: likui@caas.cn \\ Yanfang Wang, email: wangyanfang@caas.cn
}

Keywords: miR-200b/a/429, insulin resistance, high-fat-diet, knockout, adipose tissue, Pathology Section

Received: March 20, $2016 \quad$ Accepted: September 12, 2016

Published: September 16, 2016

\section{ABSTRACT}

Growing evidence demonstrates the important role of microRNAs (miRs) in regulating adipogenesis, obesity and insulin resistance. The miR-200b/a/429 cluster has been functionally characterized in mammalian reproduction; however, the potential role of the miR-200 family in adipocytes is poorly understood. The aim of our study was to investigate the physiological function of miR-200b/a/429 in the regulation of whole-body metabolism in terms of the activities and targets of this cluster in adipocytes. We generated adipocyte-specific miR-200b/a/429 knockout (ASKO) mice using a Cre-loxP system in which Cre expression was driven by the aP2 promoter. The ASKO and wild type (WT) littermate were fed a chow diet (CD) or high-fat-diet (HFD), and changes in body composition, metabolic parameters, energy homeostasis, glucose tolerance and insulin sensitivity were analyzed. The miR-200b/a/429 putative target genes were predicted and validated via luciferase reporter assays. We found that the HFD-fed ASKO mice gradually gained more body weight than the WT mice due to the increased adiposity. Decreased glucose tolerance and insulin sensitivity were also observed in the HFD-fed ASKO mice. Notably, the down-regulation of lipolysis-related genes and the decreased response to CL-316,243 stimulation in the HFD-fed ASKO mice suggested that these animals exhibited impaired lipolysis. In addition, the HFD-fed ASKO mice displayed impaired energy expenditure, indicating that the miR-200b/a/429 cluster is essential for developing adaptive responses to stressors such as HFD. For the first time, our studies demonstrated the essential role of miR-200b/a/429 in adipocytes in the regulation of HFD-induced whole-body metabolic changes.

\section{INTRODUCTION}

Adipose tissue is a fat storage depot and an endocrine organ that regulates whole-body fatty acid homeostasis [1]. There are two functionally distinct adipose tissues in mammals, white adipose tissue (WAT) and brown adipose tissue (BAT) [2], and the location, morphology and function are distinct for WAT and BAT.
Adipose tissue dysfunction is the primary cause of obesity and obesity-related metabolic disorders such as insulin resistance, diabetes and cardiovascular disease [3].

The enrichment of miR-1, miR-206 and miR-133 in BAT suggests distinct miRs profiles between WAT and BAT [4]. Robust evidence has demonstrated the role of miRs in adipogenesis, adipocyte differentiation $[5,6]$ and in the browning of white adipocytes $[7,8]$. Growing 
evidence shows that miRs are involved in obesity and obesity-related metabolic disorders [9], such as miR-223 [10], miR-378 [11], and miR-103/107 [12].

The highly conserved miR-200 family is composed of five members, miR-200a, miR-200b, miR-200c, miR141 and miR-429, which are similar in sequence and are clustered and expressed as two separate polycistronic pri-miR transcripts: the miR-200b/a/429 cluster on chromosome 4 and the miR-200c/141 cluster on chromosome 6 . The miR-200 family has been reported to be strongly expressed in lung, kidney and thymus tissues [13]. Previous studies showed the critical functions of miR-200 family in female reproduction $[14,15]$. Hasuwa et al revealed the requirement of miR-200 family and their target zeb1 in hypothalamo-pituitary-ovarian axis to support female ovulation [13]. The miR-200 family has also been shown to be involved in the epithelial-tomesenchymal transition in humans, thereby enhancing cancer cell colonization in distant tissues by targeting zeb1, and in the regulation of olfactory neurogenesis and osmotic stress in zebrafish $[16,17]$. miR-8, the homolog of miR-200 in Drosophila, has been reported to positively regulate body size [18]. Recent studies have provided evidence that miR-200b has a role in beta cell apoptosis in vitro and in vivo $[19,20]$. MiR-200, down-regulated in the livers of $\mathrm{db} / \mathrm{db}$ mice, also contribute to IL-6induced insulin resistance [21]. In hypothalamus, miR- 200a inhibition of ob/ob mice reduced body weight and improved whole-body insulin sensitivity [22]. However, the role of the miR-200b/a/429 cluster in adipose tissue is currently poorly understood.

To study the function of miR-200b/a/429 in mammalian energy homeostasis, we generated adipose tissue-specific miR-200b/a/429 knockout (ASKO) mice and revealed the critical role of this $\mathrm{miR}$ in modulating lipolysis and energy expenditure, consequently regulating obesity during periods of dietary stress. Thus, the miR$200 \mathrm{~b} / \mathrm{a} / 429$ cluster may serve as a potential target for therapeutic intervention in obesity and metabolic syndrome.

\section{RESULTS}

\section{Expression pattern of miR-200b/a/429 in adipose tissue and generation of the ASKO mice}

To detect the miR-200b/a/429 expression levels in metabolic tissues, the abundance of miR-200b/a/429 in inguinal fat (Ing-fat), gonadal fat (Gon-fat), BAT and liver were measured by real-time PCR. Our data showed that the miR-200b/a/429 cluster was most strongly expressed in Gon-fat and least strongly expressed in BAT (Figure 1A).

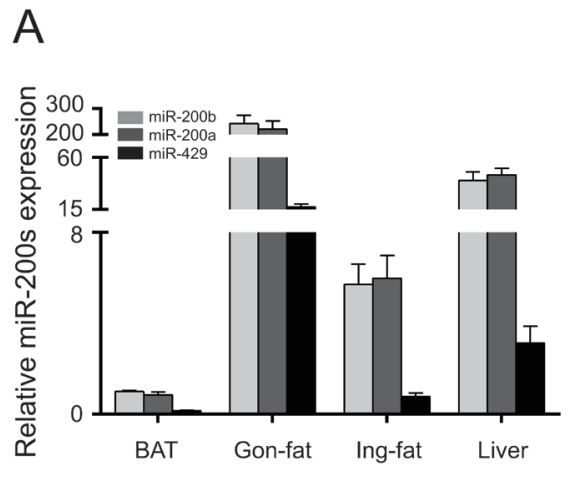

B

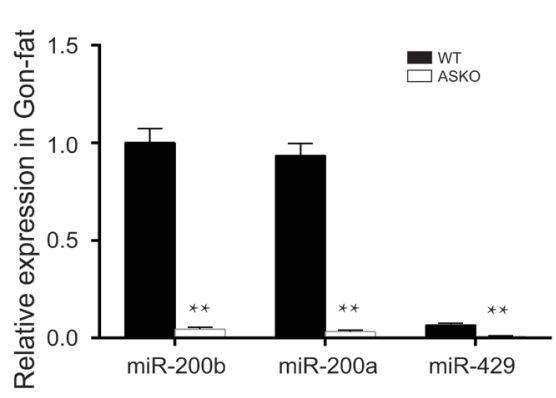

C
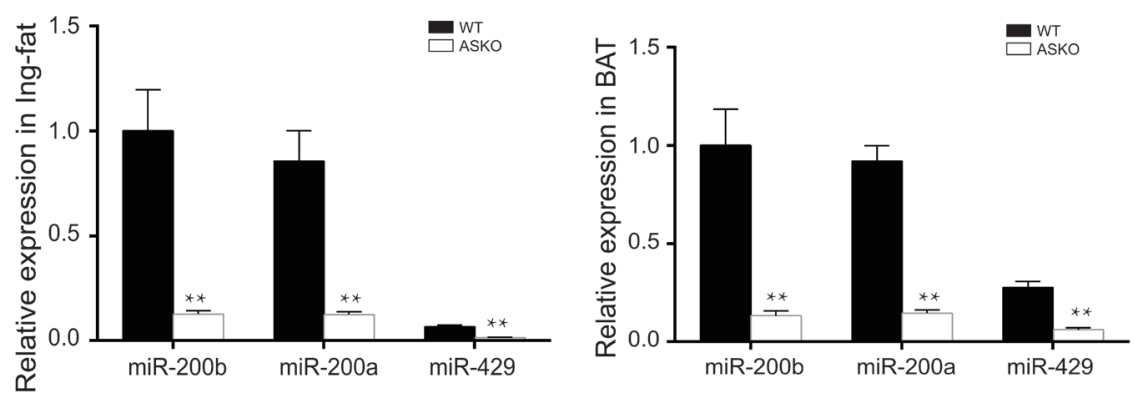

Figure 1: Expression pattern of miR-200b/a/429 in adipose tissue. A. Relative miR-200b/a/429 expression in BAT, Gon-fat, Ing-fat of WT mice $(n=4)$. Light gray bars, miR-200b; dark gray bars, miR-200a; black bars, miR-429. B.-D. MiR-200b/a/429 expression in the Gon-fat B., the Ing-fat C. and the BAT D. of 15-week-old ASKO and WT mice $(n=6)$. All of the results are presented as the means $\pm \mathrm{SEM} ; *<0.05$ and $* * p<0.01$. 
Table 1: Metabolic parameters of WT and ASKO mice fed a chow and high-fat diet

\begin{tabular}{|l|l|l|l|l|}
\hline \multirow{2}{*}{} & CD & \multicolumn{2}{l|}{ HFD } & ASKO \\
\cline { 2 - 5 } & WT & ASKO & WT & $102.53 \pm 9.64^{*}$ \\
\hline Plasma triglycerides $(\mathrm{mg} / \mathrm{dl})$ & $47.20 \pm 3.33$ & $51.50 \pm 3.72$ & $79.54 \pm 3.38$ & $108.27 \pm 4.84 * *$ \\
\hline Plasma cholesterol $(\mathrm{mg} / \mathrm{dl})$ & $69.44 \pm 5.32$ & $59.00 \pm 3.22$ & $129.68 \pm 5.33$ & $0.59 \pm 0.02$ \\
\hline Plasma NEFA $(\mathrm{mEq} / \mathrm{L})$ & $0.89 \pm 0.08$ & $0.88 \pm 0.09$ & $0.66 \pm 0.05$ & $362.56 \pm 18.70$ \\
\hline Plasma glycerol $(\mathrm{mmol} / \mathrm{L})$ & $349.68 \pm 12.34$ & $343.65 \pm 22.93$ & $365.67 \pm 11.83$ & $44.57 \pm 3.37$ \\
\hline Plasma leptin $(\mathrm{ng} / \mathrm{ml})$ & $1.74 \pm 0.20$ & $1.71 \pm 0.14$ & $40.74 \pm 2.49$ & $13.17 \pm 1.73$ \\
\hline Plasma adiponectin $(\mu \mathrm{g} / \mathrm{L})$ & $18.75 \pm 2.09$ & $16.95 \pm 2.52$ & $14.42 \pm 0.93$ & $1.81 \pm 0.51 *$ \\
\hline Plasma insulin $(\mathrm{ng} / \mathrm{ml})$ & $0.57 \pm 0.10$ & $0.30 \pm 0.02 *$ & $3.80 \pm 0.78$ & \\
\hline
\end{tabular}

Data are expressed as mean \pm SEM. 16 hours fasting. $* P<0.05, * * P<0.01 . n=7-8$ mice.

The generation of a floxed miR-200b/a/429 mouse model was described in the Supplemental Methods, and the targeting construct was showed in Supplemental Figure 1A. To obtain mice in which $\mathrm{miR}-200 \mathrm{~b} / \mathrm{a} / 429$ cluster deletion was restricted to adipose tissue, the $\mathrm{miR}^{\text {flox/flox }}$ mice were crossed with transgenic mice expressing Cre recombinase driven by the Fabp4/aP2 promoter (Supplemental Figure 1A). Genomic DNA was extracted from tails and PCR was performed using specific primers to determine the genotypes (Supplemental Figure 1B). To ascertain the miR-200b/a/429 allele status, adipose tissue was collected and the expression level of miR-200b/a/429 was detected. Our results showed that the $\mathrm{Cre}^{+} / \mathrm{miR}^{\text {flox/flox }}$ mice displayed significantly lower expression levels of miR-200b/a/429 in gonadal fat (Gonfat, Figure 1B), inguinal fat (Ing-fat, Figure 1C), and BAT (Figure 1D) than the control ( $\left.\mathrm{Cre}^{-} / \mathrm{miR}^{\text {flox/flox}}\right)$ littermates (hereafter designated as WT). The abundance of miR200b/a/429 in liver, kidney and lung tissues was not altered (Supplemental Figure 1C-1E). The ASKO mice were born at the expected Mendelian frequencies and were indistinguishable from the WT littermates.

\section{The HFD-fed ASKO mice exhibited increased body weight and adiposity}

As shown in Figure 2A, the ASKO mice displayed a similar growth rate to the WT mice when fed the CD (Figure 2A). However, when fed the HFD for 15 weeks, the ASKO mice exhibited an increased body weight, and the difference reached significance after 9 weeks of HFD induction (Figure 2A). Figure 2B shows a representative photograph of the ASKO and WT mice, and the ASKO mice weighed approximately $9.2 \%$ more than the WT mice after 15 weeks of HFD feeding (Supplemental Figure 2A).

Next, to elucidate the underlying basis for this increase in body weight, we weighed inguinal Ingfat, Gon-fat), perinephric fat (Per-fat), BAT and other organs from the ASKO and WT mice under both diet conditions. Our data revealed no difference in the weight of any fat tissue (Figure 2C-2D) or other peripheral organ (Supplemental Figure 2B) under CD conditions between the two genotypes mice. In contrast, all fat pads from the HFD-fed ASKO mice weighed more than the fat pads from the WT mice (Figure 2E-2F). All fat pads were collected together and weighted under HFD condition. Compared to the total weight of adipose tissue from WT mice, those from the ASKO mice are significantly increased with $34.6 \%$. No difference was observed in the weight of other peripheral organs under HFD conditions (Supplemental Figure 2C). Taken together, our data suggested that miR-200b/a/429 deletion from adipocytes leads to a higher body weight due to increased adiposity under HFD conditions.

Furthermore, to determine whether the increased amount of fat in ASKO mice is due to larger fat cells, the histomorphology of the adipose cells from Gonfat and BAT were analyzed. HE staining of the Gon-fat revealed the presence of enlarged adipocytes in the HFDfed ASKO mice, whereas no difference in the adipocyte size was observed in the CD-fed mice (Figure 2G). This observation was further confirmed by a $26.5 \%$ increase in the average area of individual white adipocytes from the HFD-fed ASKO mice (Figure 2H). Interestingly, adipocyte hypertrophy was not observed in the BAT from the HFDfed ASKO mice (Supplemental Figure 2D), suggesting a weak response of the BAT to HFD induction. These results indicated that the adipose tissue expansion observed in the ASKO mice is due to adipocyte hypertrophy.

\section{HFD-fed ASKO mice exhibit impaired insulin sensitivity}

To test whether the increased fat weight alters insulin sensitivity in mutant mice, glucose metabolism was assessed under both diet conditions. As shown in Figure $3 \mathrm{~A}$ and $3 \mathrm{~B}$, the response of blood glucose to exogenous glucose and insulin administration were quite similar between the CD-fed WT and ASKO mice (Figure 3A-3B). In contrast, under HFD conditions, marked phenotypic changes in glucose and insulin homeostasis were observed 
A
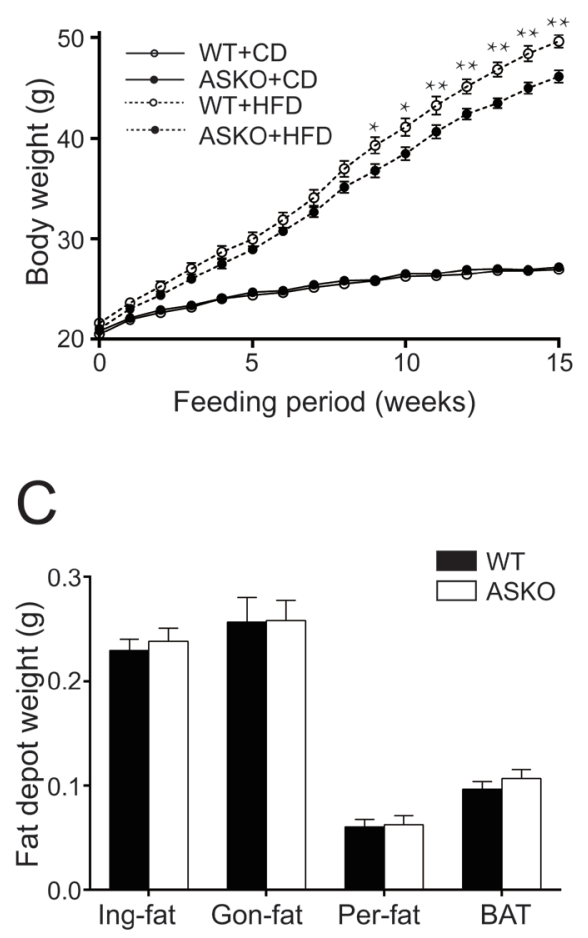

$E$

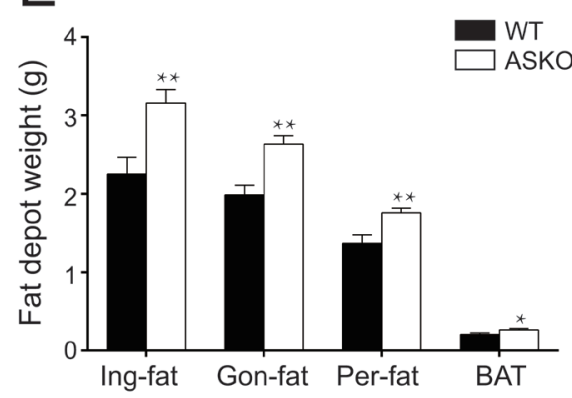

G

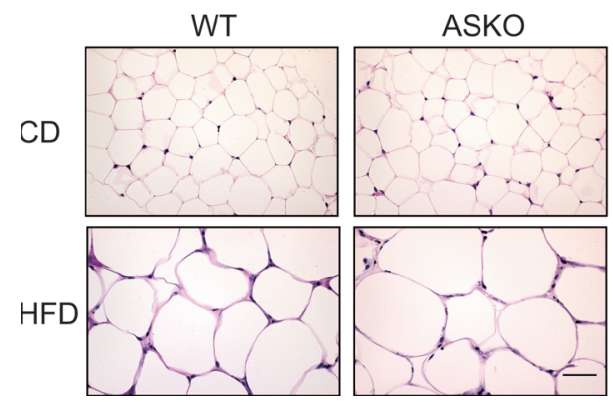

$\mathrm{B}$

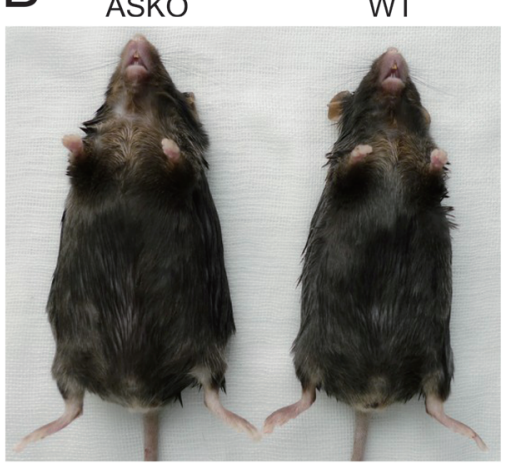

$\mathrm{D}$

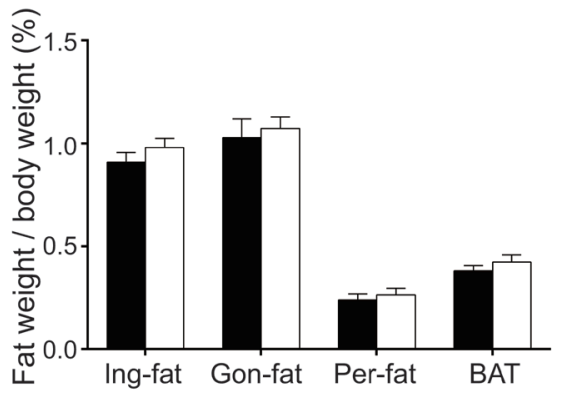

$\mathrm{F}$

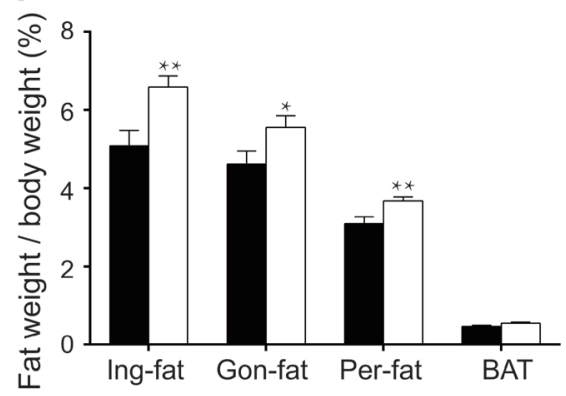

$\mathrm{H}$

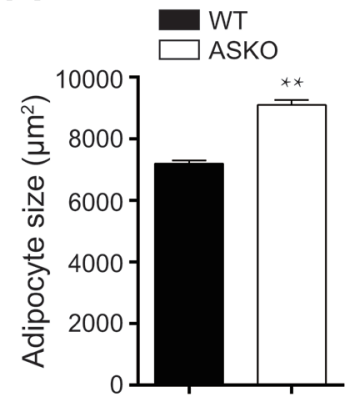

Figure 2: Increased body weight and fat content in ASKO mice under HFD conditions. A. Body weight of the WT and ASKO mice on both diet conditions $(n \geq 10)$. B. Representative photographs of mice exhibiting HFD-induced obesity. C.-D. Organ weight analysis from CD-fed D.-F., $n=12$ ) and HFD-fed E.-F., $n \geq 11$ ) mice, including absolute fat pad weight, the percentage of the total body weight and absolute weight of other peripheral organs, as indicated in the figure. G. H\&E staining of the Gon-fat from ASKO and WT mice under both diets conditions. Scale bar, $50 \mu \mathrm{m}$. H. The mean adipocyte area in the gonadal fat (square micrometers) of the HFD-fed mice. The values represent the means \pm SEM. $* p<0.05 ; * * p<0.01$. 
in the ASKO mice. The GTT assay suggested that the HFD-fed ASKO mice exhibited glucose intolerance (Figure 3C), and the significantly elevated blood glucose content upon insulin administration indicated decreased insulin sensitivity in the HFD-fed ASKO mice (Figure 3D).

Interestingly, the fasting insulin levels were significantly lower in the ASKO mice than in the WT mice under both $\mathrm{CD}$ and HFD conditions (Table 1). Additionally, decreased plasma adiponectin levels were observed in the ASKO mice compared to the WT mice, although this difference did not reach significance (Table 1). Taken together, these results strongly suggest that reduced adipose tissue insulin sensitivity results from decreased insulin levels and insulin sensitivity.

\section{Lipolysis is impaired in the HFD-fed ASKO mice}

To elucidate the potential molecular changes in adipose tissue that could account for the observed phenotypes, we examined the expression of key genes that have been reported to be involved in lipogenesis and
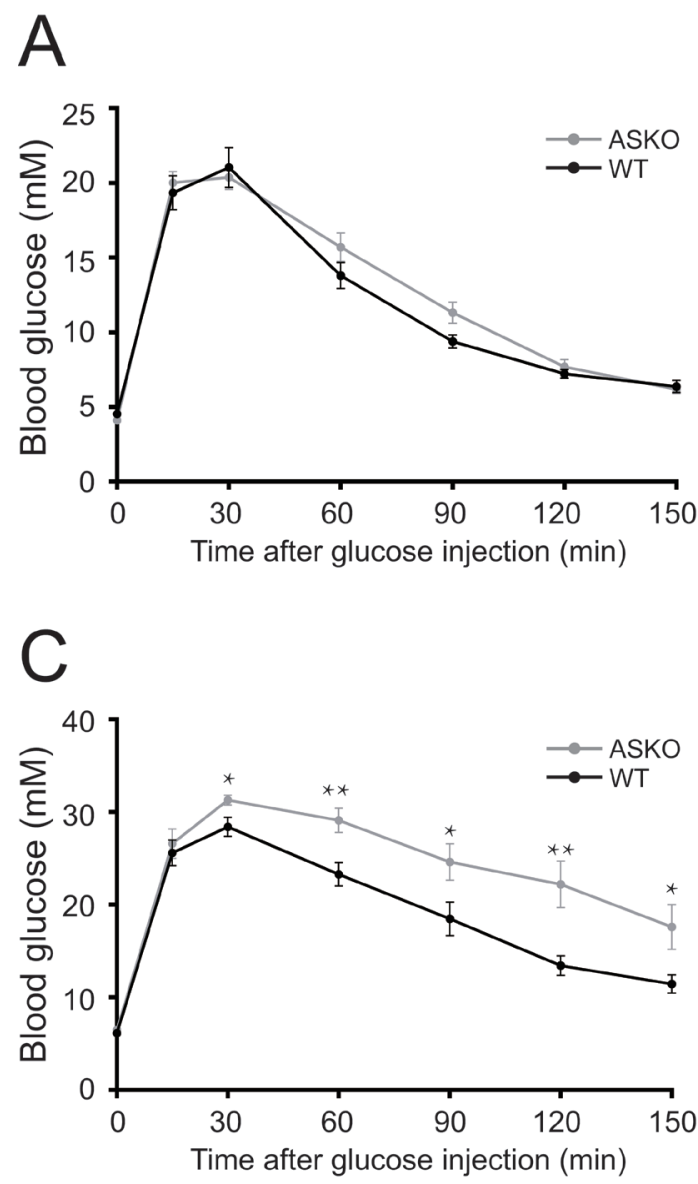

lipolysis. We found that the lipogenesis genes were not altered (Supplemental Figure 3A), suggesting that the ASKO and WT mice displayed a comparable capacity for lipid biosynthesis. However, significantly decreased levels of the $\mathrm{Hsl}$ and $\mathrm{Atgl}$ were observed in the WAT from the HFD-fed ASKO mice (Figure 4A), and the differences were further confirmed by immunoblotting (Figure 4B). Acox1 (Acyl-coenzyme A oxidase 1), the first enzyme in peroxisomal fatty acid $\beta$-oxidation, was decreased in the HFD-fed ASKO mice (Figure 4A). These data led us to speculate that lipolysis is impaired in these HFD-fed ASKO mice.

Next, we examined whether lipolysis is altered in vivo in the WAT of HFD-fed ASKO mice following stimulation with the $\beta 3$-adrenergic agonist CL-316,243. Our data showed that baseline NEFA and glycerol release was not affected by miR-200b/a/429 deficiency in adipose tissue (Figure 4C and 4D). Upon stimulation, although both the WT and ASKO mice exhibited robust lipolytic responses, significantly attenuated lipolysis was observed in the HFD-fed ASKO mice (Figure 4C and 4D).

Consistent with the reduced lipolysis in the HFDfed ASKO mice, the plasma TAG levels were significantly

$\mathrm{B}$
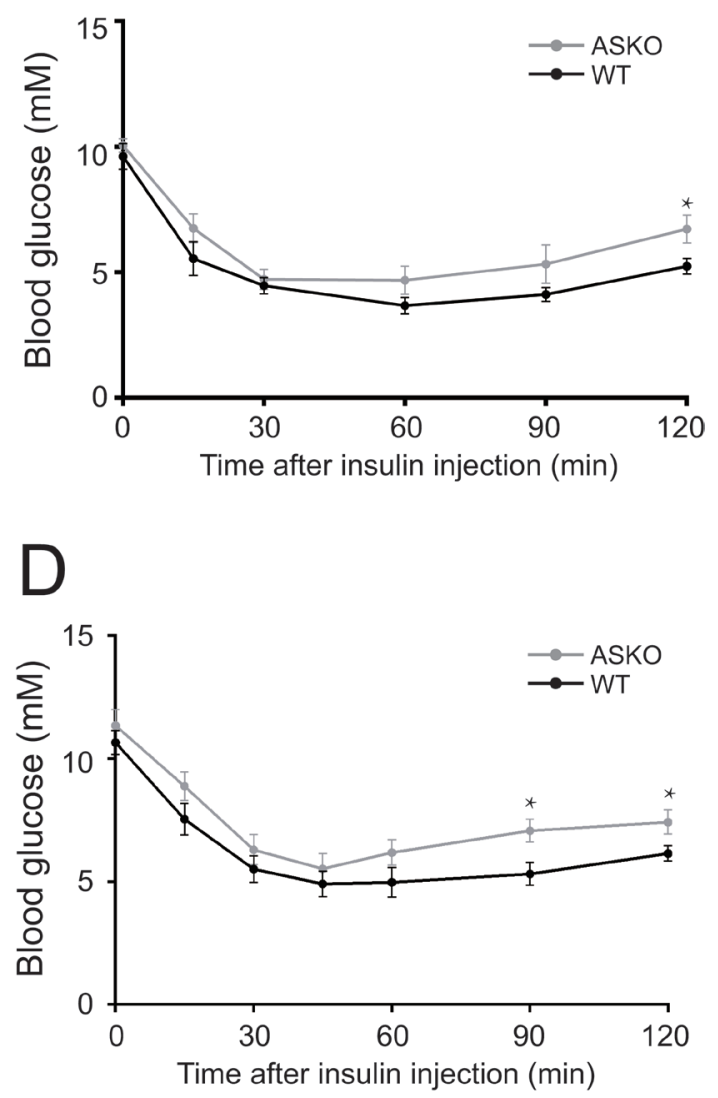

Figure 3: Impaired glucose tolerance and insulin sensitivity in the HFD-fed ASKO mice. Blood glucose levels during IPGTT A., C. and IPITT B., D. in fasted WT (black) and ASKO (gray) under CD A., B. and HFD C., D. conditions $(n=7-10)$. The values represent the means \pm SEM. ${ }^{*} p<0.05 ; * * p<0.01$. 
higher in these mice $(p<0.05)$ (Table 1$)$. Surprisingly, no significant differences in the plasma NEFA or glycerol levels were observed (Table 1). However, ASKO mice exhibited significantly lower total cholesterol levels under both diet conditions. Taken together, our data suggested that miR-200b/a/429 deficiency in adipose tissue results in reduced lipolysis, leading to a progressive increase in body weight in the ASKO mice under HFD conditions.

\section{Energy expenditureare are impaired in the ASKO mice}

To determine whether the phenotypes observed in the HFD-fed ASKO mice are related to whole-body energy consumption, the mutant and WT mice were subjected to metabolic cage studies under both diet conditions. As shown in Figure 4E-4F and Supplemental Figure 4, under CD conditions, no significant differences in the metabolic parameters of the ASKO mice were observed. In contrast, metabolic phenotyping revealed decreased energy expenditure in the HFD-fed ASKO mice, as reduced oxygen consumption (Figure 4E) and energy expenditure (Figure 4F), without any change in food intake or physiological activity (Supplemental Figure 4A-4B). A non-significant decrease in $\mathrm{CO}_{2}$ production was observed in the HFD-fed ASKO mice (Supplemental Figure $4 \mathrm{C}$ ). The RQ, which is the ratio of the amount of $\mathrm{CO}_{2}$ produced to oxygen consumed by breathing, was highly similar between the WT and ASKO mice under both diet conditions (Supplemental Figure 4D).

As BAT is a critical organ for energy homeostasis, we also measured the expression levels of genes known to be involved in BAT metabolism in the HFD-fed mice. Our data showed that the mRNA levels of these genes were not significantly altered in the HFD-fed ASKO mice (Supplemental Figure 3B).

\section{Putative metabolic targets of miR-200b}

The TargetScan prediction program was used to identify target genes containing binding sites for the miR paralog miR-200b. Four predicted target genes, epidermal growth factor receptor pathway substrate 8 (Eps 8$)$, lipoma HMGIC fusion partner (Lhfp), GLIS family zinc finger 2 (Glis2) and ribosomal protein S6 kinase, polypeptide 1 (Rps6kb1), which have been reported to be predominately associated with obesity and insulin resistance, were selected for further validation via luciferase reporter assays. The predicted miR-200b binding sites on the 3'UTRs of those four genes are shown in Figure 5A. Luciferase assays revealed the repression of the Eps 8 3'UTR S1 reporter by a miR-200b mimic, whereas this repressive effect was diminished for the Eps8 3'UTR S2 reporter and mutated reporter (Figure 5B). As shown in Figure $5 \mathrm{C}-5 \mathrm{E}$, the luciferase activities were reduced in the cells transfected with a plasmid containing the 3'UTR of Lhfp, Glis 2 or Rps6kb1 carrying miR-200b binding sites in the presence of miR-200b mimics, whereas these inhibitory effects were not observed for the nonspecific scrambled oligonucleotides (Figure 5C-5E). Furthermore, real-time PCR data revealed the significant elevation of Lhfp and Rps6kbl in the HFD-fed ASKO mice. No difference in Eps 8 and Glis 2 RNA expression was observed between the ASKO and WT mice (Figure 5F), suggesting that Eps 8 and Glis 2 genes may be posttranscriptionally regulated by miR-200b/429.

\section{DISCUSSION}

In this study, to explore the potential effect of miR$200 \mathrm{~b} / \mathrm{a} / 429$ in adipocytes on metabolic homeostasis, ASKO mice were successfully generated leaving the host gene Ttll10 intact (data not shown). The ASKO mice were born at the expected Mendelian frequencies and were indistinguishable from their WT littermates fed the CD. However, when fed the HFD, the ASKO mice developed diet-induced obesity, demonstrating that adipose miR$200 \mathrm{~b} / \mathrm{a} / 429$ does not positively regulate systemic growth of mice. It has been reported that Drosophila lacking miR-8, the sole homolog of miR-200b/a/429, exhibit a significantly smaller body size and reduced insulin signaling [18]. Our data suggest either that the role of the miR-200 family in the regulation of body weight homeostasis is not evolutionarily conserved or that mice are not completely analogous to flies. No elevation of liver fat deposition was observed based on H\&E staining analysis (Supplemental Figure 3C), we speculate that fatty liver may ultimately develop in the ASKO mice over time for the high level of serum total triglycerides.

Similar to other mouse strains carrying a global deletion of miR-378/378* [11], our ASKO mice exhibited overt phenotypes in response to excessive calorie intake. Mice lacking miR-378/378* are lean and resistant to HFD-induced obesity, whereas our ASKO mice are sensitive to diet-induced obesity. Impaired lipolysis in the WAT was observed in the ASKO mice, as indicated by the significantly down-regulated expression levels of the lipolysis-related genes and the significantly alleviated alterations in the serum FA and glycerol levels upon CL-316,243 stimulation. Surprisingly, in the absence of stimulation, no changes in the serum FA and glycerol levels of the ASKO mice were observed under either diet condition. We speculated that this lack of an effect could be due to either less severe lipolysis defects or to a global deceleration of FA turnover in ASKO mice. This finding is in agreement with previous observations in many obese individuals [23] and HSL haploinsufficient mice [24]. We also observed the significant down-regulated Acox 1 in the HFD-fed ASKO mice, which might decrease the utilization of fatty acids and ultimately lead to fat deposition. Taken together, all these observations could partially explain 
the HFD-induced obesity of ASKO mice and the detailed mechanisms still need to be further explored.

Furthermore, we found that disrupted adipose tissue homeostasis leads to glucose intolerance in the HFD-fed ASKO mice. Unexpectively, we observed the lower level of serum insulin content in ASKO mice, which might due to the dysfunction of pancreas and pancreatic islet. Taken together, our data suggested that the glucose intolerance
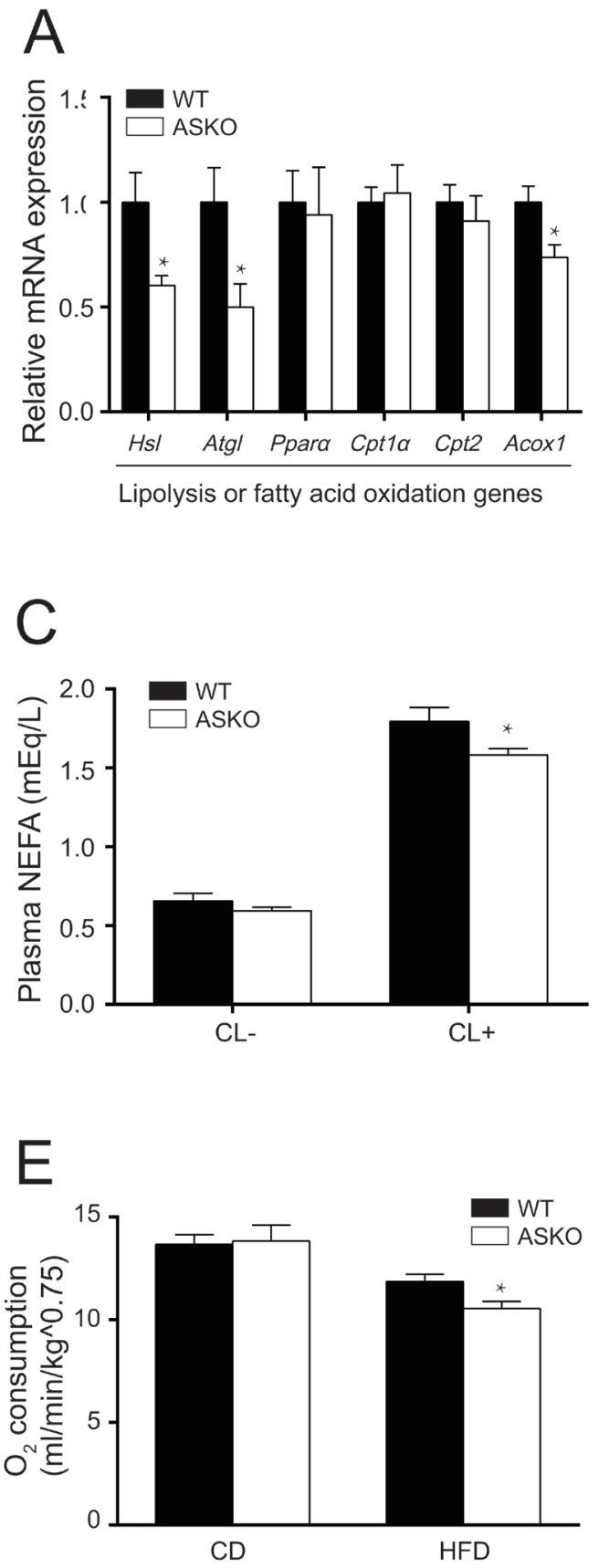

of those mice might be caused by both low serum insulin levels and insulin resistance. Normally, the low level of serum insulin content is associated with better insulin sensitivity, the opposite observation in our study might due to the failure of the signaling pathways of IRS-PI3KAkt, as miR-200b has been reported to be involved in this pathway $[18,25,26]$. In this regard, the precise role of the miR-200b/a/429 cluster on the signaling pathways of IRS-
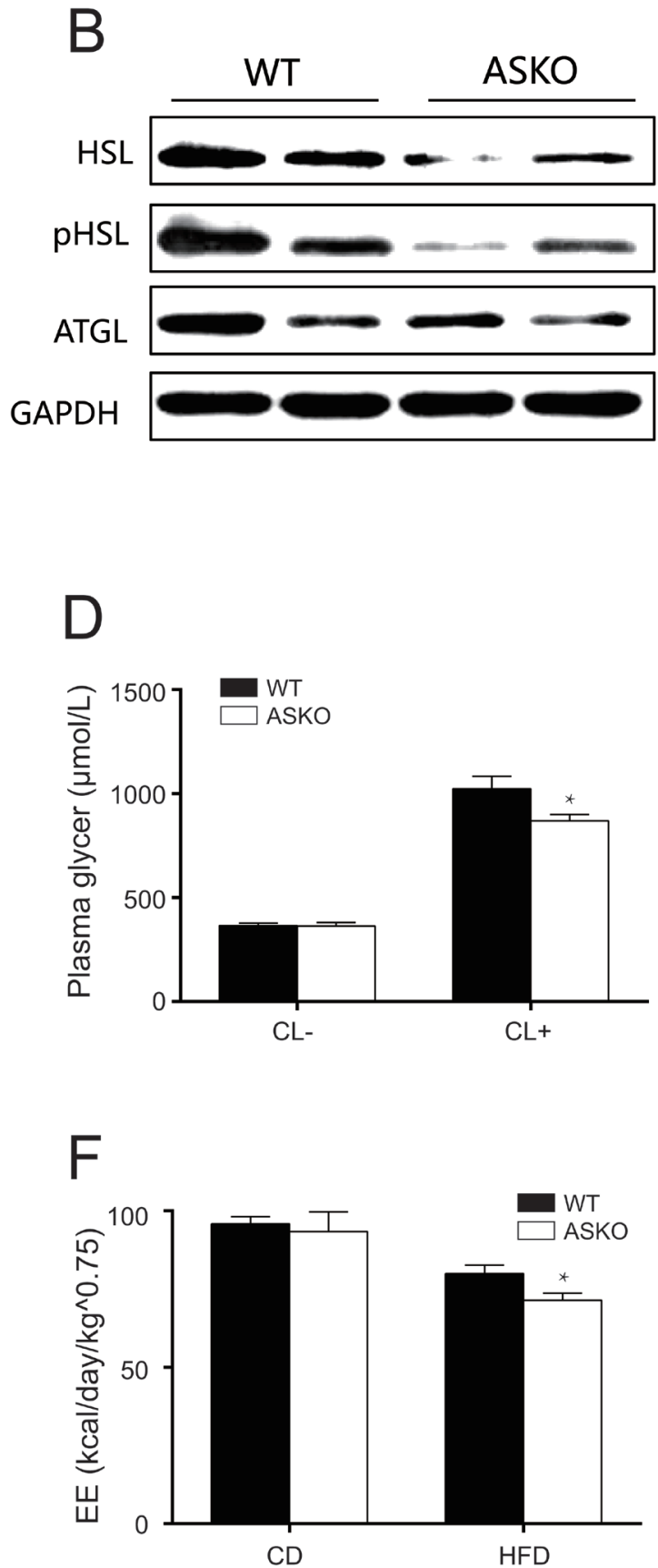

Figure 4: Ablation of miR-200b/a/429 reduces lipolysis and energy expenditure in vivo. A. The mRNA expression of lipolysis-related genes in Gon-fat from HFD-fed WT and ASKO mice $(n=6)$. B. Representative immunoblotting for HSL, pHSL, ATGL in Gon-fat from HFD-fed mice. C., D. The circulating levels of NEFA C. and glycerol D. on $\beta 3$-AR agonist CL-316, 243 stimulation $(n=$ 5-8). E. The O2 consumption and energy expenditure (EE) F. of male WT and ASKO mice was monitored during feeding with the CD or the HFD $(n=5-7)$. The values represent the means \pm SEM. ${ }^{*} p<0.05 ; * * p<0.01$. 


\section{A}

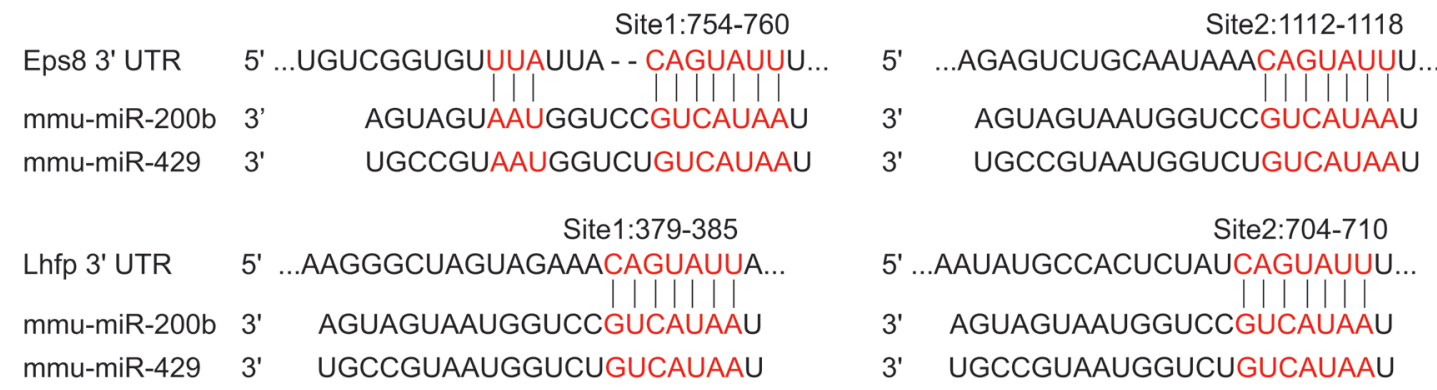

Site1:1580-1586

Glis2 3' UTR 5' ...GCACAUUCCAGGAUUCAGUAUUU...

mmu-miR-200b 3' AGUAGUAAUGGUCC - - GUCAUAAU

mmu-miR-429 3' UGCCGUAAUGGUCU - - GUCAUAAU

Site1:317-323

Rps6kb1 3' UTR 5' ...AUGGCUGCAAACUGACAGUAUUA...

mmu-miR-200b 3' AGUAGUAAUGGUCCGUCAUAAU

mmu-miR-429 $\quad$ 3' UGCCGUAAUGGUCUGUCAUAAU
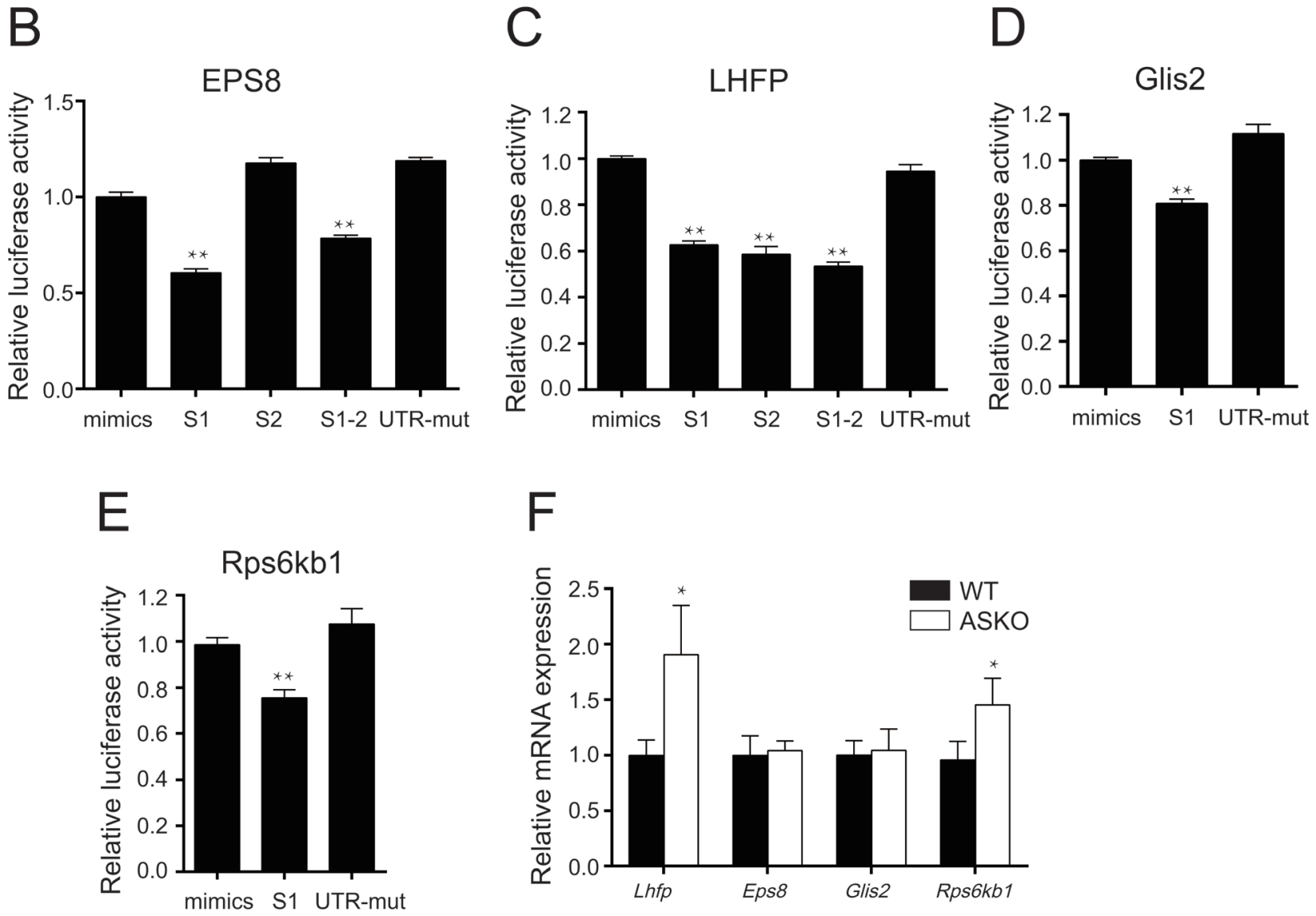

Figure 5: miR-200b targets the 3'UTRs of Eps8, Lhfp, Glis 2 and Rps6kb1. A. TargetScan identified the conserved predicted miR-200b binding sites on the 3'UTRs of Eps8, Lhfp, Glis2 and Rps6kb1. B.-E. Quantification of the dual-luciferase assay in HEK293 cells co-transfected with an miR-200 mimic or control oligonucleotides and the Eps 8 B., Lhfp C., Glis2 D., Rps6kb1 E. or mutant 3'UTR reporter plasmid $(n=4)$. F. mRNA expression of Eps8, Lhfp, Glis2 and Rps6kb1 genes in Gon-fat of the WT (black bars) and ASKO (white bars) mice $(n=6)$. mut, mutant. The values are expressed as the means \pm SEM. $* p<0.05, * * p<0.01$. 
PI3K-Akt in the target organs of insulin resistance must be further explored. It has been established that many miRs exert regulatory effects on insulin signaling and glucose metabolism at multiple levels [9, 27, 28]. Trajkovski et al. overexpressed miR-107 predominantly in mouse adipose tissue via adenovirus injection and observed impaired glucose tolerance and insulin sensitivity [12]. It is of interest to investigate whether the adenoviral injection of miR-200b/a/429 into the adipose tissue of HFD-induced ASKO mice restores insulin signaling.

It is well known that obesity is generally caused by an imbalance between energy intake and energy expenditure $[29,30,31]$. In this study, metabolic phenotyping analysis revealed that the energy balance was altered in the HFD-fed ASKO mice. Consistent with our finding, impaired energy expenditure was observed in mice lacking miR-378/378* [11] and in miR-196 transgenic mice [32].

MiRs modulate complex physiological and pathological pathways via multiple target networks. The miR-200b/a/429 complex undoubtedly targets many genes. Its putative targets were predicted computationally, and luciferase reporter assays suggested that the miR200b/429 cluster targets the eps8, lhfp, glis 2 and rps6kb1 genes. According to the mutual exclusion model of miRmRNA interactions, cells in which an miR is silenced should highly express its targets [33, 34, 35]. Thus, we hypothesized that these targets were up-regulated at the protein level in the WAT of ASKO mice. In this regard, our data are in agreement with the observation that mice carrying a genetic deletion of Eps8, a regulator of actin dynamics, leads to reduced body weights and partial resistance to diet-induced obesity [36]. Similarly, it has been reported that the absence of Rps $6 \mathrm{~kb} 1$, another putative miR-200b/a/429 target, in mice protects against age- and diet-induced obesity [33]. Taken together, we propose that the miR-200b/a/429 cluster exerts its regulatory functions via its targets, such as Eps8 and Rps6kb1. However, further investigation is required to elucidate the molecular mechanisms and regulatory pathways by which these miRs alter fat metabolism in HFD-fed ASKO mice.

In summary, our data reveal the role of adipose miR200b/a/429 in HFD-induced obesity and insulin resistance, and suggest a promising strategy for addressing the health issues caused by obesity and its associated diseases.

\section{MATERIALS AND METHODS}

\section{Animals}

The mice were maintained in a C57BL/6 background, were housed in a pathogen-free facility at the Institute of Animal Science of the Chinese Academy of Agricultural Sciences (CAAS, Beijing, China) in a 12 $\mathrm{h}$ light-dark cycle. Mice were provided with free access to standard irradiated normal rodent chow diet (CD; diet 1035; HFK Bioscience, Beijing, China) for 22 weeks. All experiments involving mice were approved by the Institutional Animal Care Research Advisory Committee of the CAAS.

\section{High-fat-diet treatment}

Once mice were genotyped, they were aliquot randomly into high-fat-diet (HFD; 60\% of energy from fat; D12492; Research Diets, New Brunswick, NJ, USA) and chow diet groups. HFD was fed to $20 \mathrm{~g} \mathrm{WT}$ and ASKO male mice and the body weight was measured and recorded weekly. After 15-week of HFD induction, ASKO and WT mice were used for the following experiments and assays, including adipose tissues histological stains, the blood parameters, GTT, ITT, metabolic cages analysis and Western blot.

\section{Real-time PCR analysis}

Total RNA was isolated from mouse tissue using TRIzol (Invitrogen, Carlsbad, CA, USA) according to the manufacturer's instructions. Then, $2 \mu \mathrm{g}$ of total RNA was reverse-transcribed using the First Strand cDNA Synthesis Kit (Thermo Scientific, Waltham, MA, USA). QRT-PCR was performed using SYBR Green master mix and the 7500 Fast Real Time PCR system (Applied Biosystems, Warrington, UK). The primers used for real-time PCR are shown in Supplemental Table 1. QRT-PCR of the miRs was performed using TaqMan MicroRNA assay kits for mmu-miR-200b-3p, mmu-miR-200a-3p, mmumiR-429-3p and U6 snRNA (Applied Biosystems, Foster City, USA) according to the manufacturer's instructions. MiR expression analysis was conducted using TaqMan Universal PCR Master Mix (Applied Biosystems, Foster City, CA USA). Gene expression was normalized to U6 or Gapdh for miRs and mRNAs, respectively. Relative gene expression was calculated using the comparative cycle threshold $\left(2^{-\Delta \Delta \mathrm{Ct}}\right)$ method [37].

\section{Western blotting}

Tissues were lysed in T-PER Tissue Protein Extraction Reagent (Thermo-Fisher, Rockford, MD, USA) in the presence of a protease inhibitor cocktail (Roche, Shanghai, China). The lysates and protein markers (Fermentas, York, UK) were resolved via SDS-PAGE, transferred to PVDF membranes (Millipore, Bedford, MA, USA), and probed using anti-Hsl (cat. no. 4107, CST, Danvers, MA, USA), anti-phospho-HSL Ser563 (cat. no. 4139, CST, Danvers, MA, USA), anti-ATGL 
(cat. no. 2138, CST, Beverly, MA, USA) and anti-GAPDH antibodies (cat. no. 8884, CST, Beverly, MA, USA). The immunoreactive bands were detected using Pierce ECL Western Blotting Substrate (Thermo, Waltham, MA, USA). The protocol was described in our previous study [38].

\section{Metabolic analyses}

The HFD-fed and CD-fed mice ( $n=5-7)$ were housed in individual metabolic chambers and were allowed to acclimatize for $24 \mathrm{~h}$ before data collection. Energy expenditure of these mice was measured using an indirect open-circuit calorimeter (Oxylet, Panlab, Barcelona, Spain). Data on $\mathrm{O}_{2}$ consumption $\left(\mathrm{VO}_{2} ; \mathrm{ml} / \mathrm{min} /\right.$ $\left.\mathrm{kg}^{\wedge} 0.75\right)$ and $\mathrm{CO}_{2}$ production $\left(\mathrm{VCO}_{2} ; \mathrm{ml} / \mathrm{min} / \mathrm{kg}^{\wedge} 0.75\right)$ were collected every $30 \mathrm{~min}$. Locomotor activity was measured by continuously recording spontaneous activity using extensiometric weight transducers placed below the cages, which enabled the detection of activity even without displacement. The respiratory quotient (RQ) was calculated as the ratio of $\mathrm{VO}_{2}$ to $\mathrm{VCO}_{2}$, and energy expenditure was determined as $(3.815+1.232 \times \mathrm{RQ}) \times$ $\mathrm{VO}_{2}$ and was expressed as $\mathrm{kcal} / \mathrm{h} /$ mouse [39]. The data is normalized to body weight.

\section{Glucose and insulin tolerance tests}

We measured the blood glucose concentration using a glucose meter (OneTouch, Milpitas, CA, USA) for the intraperitoneal glucose tolerance test (IPGTT) and the intraperitoneal insulin tolerance test (IPITT) $(n=7-10)$. We performed the IPGTT after a 16-h fast using an i.p. injection of $2 \mathrm{~g}$ of glucose/ $\mathrm{kg}$ body weight. For the IPITT, the fasting blood glucose level was measured (4-h fast, blood collected from the tail vein). Then, insulin was injected i.p. (0.75 U/kg body weight, Humulin, Eli Lilly, Indianapolis, IN, USA), and blood samples were collected immediately before (time 0 ) and at 30, 60, 90, 120 and 150 min after insulin injection for glucose measurement.

\section{Blood analysis}

Blood was obtained via the caudal vein before the mice $(n=7-8)$ were sacrificed. The TAG and cholesterol contents were measured using enzymatic assay kits from Applygen (Beijing, China). The plasma insulin, leptin and adiponectin levels were measured via ELISA (Millipore, Bedford, MA, USA), and the free glycerol content (Sigma, St. Louis, MO, USA) and the nonesterified fatty acid (NEFA) levels were measured via a colorimetric assay (Wako Chemical, Osaka, Japan).

\section{Histological studies}

The tissues from HFD- and CD- diet mice with different genotypes $(n=4)$ were dissected, fixed in $4 \%$ paraformaldehyde overnight and rinsed with phosphatebuffered saline, followed by embedding in paraffin. The paraffin-embedded WAT, BAT and liver tissues were sectioned at a thickness of $5 \mu \mathrm{m}$ and stained with hematoxylin and eosin (H\&E). The adipocyte size of Gonfat from HFD-fed WT and ASKO mice were measured by using Image J software as previously described [40]. Adipocyte size was determined on sections and measured in five different sections from each mice fat pad (four mice per group), after which the area of 50 cells in each section were measured.

\section{Lipolysis}

For in vivo lipolysis assay, HFD-fed WT and ASKO mice were fasted for 4 hours and injected i.p. with the $\beta 3$-specific agonist CL-316,243 (CL, $0.1 \mathrm{mg} / \mathrm{kg}$, Sigma, MO, USA). Blood was collected before and 5 min after injection for the measurement of the NEFA and glycerol levels.

\section{Luciferase reporter constructs and assay}

The 3'UTR portions of the mouse Eps 8 , Lhfp, Glis 2 and Rps $6 \mathrm{~kb} 1 \mathrm{mRNAs}$ were cloned into the psiCHECK-2 vector (Promega, Madison, WI, USA). The 3'UTR portions of target genes not corresponding to a target sequence were used as controls. The specific primers are listed in Supplemental Table 1. Then, $100 \mathrm{ng}$ of each psiCHECK-2 construct was co-transfected with $50 \mathrm{nM}$ miR mimics or a negative control into HEK293 cells in a 24-well plate using Lipofectamine 2000 (Invitrogen, Carlsbad, CA, USA). HEK293 cell lines were maintained in DMEM supplemented with $10 \% \mathrm{FBS}$ at $37{ }^{\circ} \mathrm{C}$ in a $5 \%$ $\mathrm{CO}_{2}$ environment. After 48 hours, the cell extracts were obtained, and the firefly and Renilla luciferase activity levels were measured using the Dual-Luciferase reporter system (Promega, Madison, WI, USA) according to the manufacturer's instructions.

\section{Statistical analysis}

The data are presented as the means \pm s.e.m. The values were analyzed via two-tailed independent sample Student's t tests, using GraphPad Prism version 6 (GraphPad Software, La Jolla, CA, USA). A value of $p<$ 0.05 was considered to be significant. 


\section{ACKNOWLEDGMENTS}

We thank Gaoxiang's laboratory of the Model Animal Research Center of Nanjing University for their helpful support of generating $\mathrm{miR}^{\text {flox/flox }}$ mice. This work was supported by the National Natural Science Foundation of China (grant numbers 31330074 and 31101688), the State Key Development Program for Basic Research of China (grant number 2015CB943100), the Agricultural Science and Technology Innovation program (ASTIPIAS05), and Y. Wang was supported by the Elite Youth Program of the Chinese Academy of Agricultural Sciences.

\section{CONFLICTS OF INTEREST}

The authors declare no financial or commercial conflicts of interest.

\section{REFERENCES}

1. Galic S, Oakhill JS, Steinberg GR. Adipose tissue as an endocrine organ. Mol Cell Endocrinol. 2010; 316: 129-139.

2. Cannon B, Nedergaard J. Brown Adipose Tissue: Function and Physiological Significance. Physiol Rev. 2004; 84: 277 359.

3. Blüher M. Adipose tissue dysfunction in obesity. Exp Clin Endocrinol Diabetes. 2009; 117: 241-250.

4. Walden TB, Timmons JA, Keller P, Nedergaard J, Cannon B. Distinct expression of muscle-specific microRNAs (myomirs) in brown adipocytes. J Cell Physiol. 2009; 218: 444-9.

5. Mudhasani R, Puri V, Hoover K, Czech MP, Imbalzano $\mathrm{AN}$, Jones SN. Dicer is required for the formation of white but not brown adipose tissue. J Cell Physiol. 2011; 226: 1399-406.

6. Kim H-J, Cho H, Alexander R, Patterson HC, Gu M, Lo KA, Xu D, Goh VJ, Nguyen LN, Chai X, Huang CX, Kovalik J-P, Ghosh S, et al. MicroRNAs Are Required for the Feature Maintenance and Differentiation of Brown Adipocytes. Diabetes. 2014; 63: 4045-56.

7. Mori M, Nakagami H, Rodriguez-Araujo G, Nimura K, Kaneda Y. Essential role for miR-196a in brown adipogenesis of white fat progenitor cells. PLoS Biol. 2012; 10: e1001314.

8. Liu W, Bi P, Shan T, Yang X, Yin H, Wang Y-X, Liu N, Rudnicki MA, Kuang S. miR-133a regulates adipocyte browning in vivo. PLoS Genet. 2013; 9: e1003626.

9. Rottiers V, Näär AM. MicroRNAs in metabolism and metabolic disorders. Nat Rev Mol Cell Biol. 2012; 13: 23950 .

10. Zhuang G, Meng C, Guo X, Cheruku PS, Shi L, Xu H, Li H, Wang G, Evans AR, Safe S. A Novel Regulator of Macrophage Activation miR-223 in Obesity-Associated Adipose Tissue Inflammation. Circulation. 2012; 125:
2892-2903.

11. Carrer M, Liu N, Grueter CE, Williams AH, Frisard MI, Hulver MW, Bassel-Duby R, Olson EN. Control of mitochondrial metabolism and systemic energy homeostasis by microRNAs 378 and 378*. Proc Natl Acad Sci. 2012; 109: 15330-15335.

12. Trajkovski M, Hausser J, Soutschek J, Bhat B, Akin A, Zavolan M, Heim MH, Stoffel M. MicroRNAs 103 and 107 regulate insulin sensitivity. Nature. 2011; 474: 649-53.

13. Hasuwa H, Ueda J, Ikawa M, Okabe M. miR-200b and miR-429 function in mouse ovulation and are essential for female fertility. Science. 2013; 341: 71-73.

14. Renthal NE, Chen C-C, Koriand'r CW, Gerard RD, PrangeKiel J, Mendelson CR. miR-200 family and targets, ZEB1 and ZEB2, modulate uterine quiescence and contractility during pregnancy and labor. Proc Natl Acad Sci. 2010; 107: 20828-20833.

15. Koriand'r CW, Renthal NE, Condon JC, Gerard RD, Mendelson CR. MicroRNA-200a serves a key role in the decline of progesterone receptor function leading to term and preterm labor. Proc Natl Acad Sci. 2012; 109: 75297534.

16. Flynt AS, Thatcher EJ, Burkewitz K, Li N, Liu Y, Patton JG. miR-8 microRNAs regulate the response to osmotic stress in zebrafish embryos. J Cell Biol. 2009; 185: 115127.

17. Thiery JP, Acloque H, Huang RY, Nieto MA. Epithelialmesenchymal transitions in development and disease. Cell. 2009; 139: 871-890.

18. Hyun S, Lee JH, Jin H, Nam J, Namkoong B, Lee G, Chung J, Kim VN. Conserved MicroRNA miR-8/miR-200 and its target USH/FOG2 control growth by regulating PI3K. Cell. 2009; 139: 1096-1108.

19. Filios SR, Xu G, Chen J, Hong K, Jing G, Shalev A. MicroRNA-200 is induced by thioredoxin-interacting protein and regulates Zeb1 protein signaling and beta cell apoptosis. J Biol Chem. 2014; 289: 36275-83.

20. Belgardt B-F, Ahmed K, Spranger M, Latreille M, Denzler R, Kondratiuk N, von Meyenn F, Villena FN, Herrmanns K, Bosco D, Kerr-Conte J, Pattou F, Rülicke T, et al. The microRNA-200 family regulates pancreatic beta cell survival in type 2 diabetes. Nat Med. 2015; 21: 619-27.

21. Dou L, Zhao T, Wang L, Huang X, Jiao J, Gao D, Zhang H, Shen T, Man Y, Wang S, Li J. miR-200s Contribute to Interleukin-6 (IL-6)-induced Insulin Resistance in Hepatocytes. J Biol Chem. 2013; 288: 22596-606.

22. Crépin D, Benomar $\mathrm{Y}$, Riffault L, Amine H, Gertler A, Taouis M. The over-expression of miR-200a in the hypothalamus of ob/ob mice is linked to leptin and insulin signaling impairment. Mol Cell Endocrinol. 2014; 384: $1-11$.

23. Karpe F, Dickmann JR, Frayn KN. Fatty acids, obesity, and insulin resistance: time for a reevaluation. Diabetes. 2011; 60: 2441-9. 
24. Girousse A, Tavernier G, Valle C, Moro C, Mejhert N, Dinel A-L, Houssier M, Roussel B, Besse-Patin A, Combes M, Mir L, Monbrun L, Bézaire V, et al. Partial Inhibition of Adipose Tissue Lipolysis Improves Glucose Metabolism and Insulin Sensitivity Without Alteration of Fat Mass. PLoS Biol. 2013; 11: e1001485.

25. Liu G-T, Chen H-T, Tsou H-K, Tan T-W, Fong Y-C, Chen P-C, Yang W-H, Wang S-W, Chen J-C, Tang C-H. CCL5 promotes VEGF-dependent angiogenesis by downregulating miR-200b through $\mathrm{PI} 3 \mathrm{~K} / \mathrm{Akt}$ signaling pathway in human chondrosarcoma cells. Oncotarget. 2014; 5: 10718-31. doi: 10.18632/oncotarget.2532.

26. Xiao Y, Wang J, Chen Y, Zhou K, Wen J, Wang Y, Zhou Y, Pan W, Cai W. Up-regulation of miR-200b in biliary atresia patients accelerates proliferation and migration of hepatic stallate cells by activating PI3K/Akt signaling. Cell Signal. 2014; 26: 925-32.

27. Lynn FC. Meta-regulation: microRNA regulation of glucose and lipid metabolism. Trends Endocrinol Metab. 2009; 20: 452-9.

28. Dumortier O, Hinault C, Van Obberghen E. MicroRNAs and Metabolism Crosstalk in Energy Homeostasis. Cell Metab. 2013; 18: 312-24.

29. Martinez JA. Body-weight regulation: causes of obesity. Proc Nutr Soc. 2000; 59: 337-345.

30. Crowley VEF, Yeo GSH, O'Rahilly S. Obesity therapy: altering the energy intake-and-expenditure balance sheet. Nat Rev Drug Discov. 2002; 1: 276-86.

31. Swinburn B, Ravussin E. Energy balance or fat balance? Am J Clin Nutr. 1993; 57: 766S-770S.

32. Mori M, Nakagami H, Rodriguez-Araujo G, Nimura K, Kaneda Y. Essential role for miR-196a in brown adipogenesis of white fat progenitor cells. PLoS Biol. 2012; 10: e1001314.
33. Um SH, Frigerio F, Watanabe M, Picard F, Joaquin M, Sticker M, Fumagalli S, Allegrini PR, Kozma SC, Auwerx J, Thomas G. Absence of S6K1 protects against age- and diet-induced obesity while enhancing insulin sensitivity. Nature. 2004; 431: 200-5.

34. Um SH, D’Alessio D, Thomas G. Nutrient overload, insulin resistance, and ribosomal protein S6 kinase 1, S6K1. Cell Metab. 2006; 3: 393-402.

35. Carnevalli LS, Masuda K, Frigerio F, Le Bacquer O, Um SH, Gandin V, Topisirovic I, Sonenberg N, Thomas G, Kozma SC. S6K1 Plays a Critical Role in Early Adipocyte Differentiation. Dev Cell. 2010; 18: 763-74.

36. Tocchetti A, Ekalle Soppo CB, Zani F, Bianchi F, Gagliani MC, Pozzi B, Rozman J, Elvert R, Ehrhardt N, Rathkolb B, Moerth C, Horsch M, Fuchs H, et al. Loss of the Actin Remodeler Eps8 Causes Intestinal Defects and Improved Metabolic Status in Mice. PLoS ONE. 2010; 5: e9468.

37. VanGuilder HD, Vrana KE, Freeman WM. Twenty-five years of quantitative PCR for gene expression analysis. BioTechniques. 2008; 44: 619-26.

38. Zhang J, Ying Z, Tang Z, Long L, Li K. MicroRNA-148a Promotes Myogenic Differentiation by Targeting the ROCK1 Gene. J Biol Chem. 2012; 287: 21093-101.

39. Matsen ME, Thaler JP, Wisse BE, Guyenet SJ, Meek TH, Ogimoto K, Cubelo A, Fischer JD, Kaiyala KJ, Schwartz MW, others. In uncontrolled diabetes, thyroid hormone and sympathetic activators induce thermogenesis without increasing glucose uptake in brown adipose tissue. Am J Physiol-Endocrinol Metab. 2013; 304: E734-E746.

40. Liu X, Wang S, You Y, Meng M, Zheng Z, Dong M, Lin J, Zhao Q, Zhang C, Yuan X, Hu T, Liu L, Huang Y, et al. Brown Adipose Tissue Transplantation Reverses Obesity in Ob/Ob Mice. Endocrinology. 2015; 156: 2461-9. 